\title{
Amenaza de parto prematuro. Rotura prematura de membranas. Corioamnionitis
}

Preterm labour. Premature rupture of membranes. Chorioamnionitis

\author{
A. Ochoa, J. Pérez Dettoma
}

\section{RESUMEN}

Cuanto menor es la edad gestacional al nacer, mayor es el riesgo de morbimortalidad perinatal y de morbilidad materna (aumento del número de cesáreas, metritis postparto). Es importante diagnosticar a tiempo una amenaza de parto prematuro (APP) para comenzar con el tratamiento tocolítico y conseguir una maduración pulmonar fetal eficaz. También es necesario distinguir lo que es una verdadera de una falsa APP. Hay una alta incidencia de sobrediagnóstico y de sobretratamiento y es frecuente la hospitalización prolongada. Con las pruebas que informan sobre la modificación cervical y la dinámica uterina se puede establecer un diagnóstico certero que conlleve una conducta adecuada. La identificación temprana y la prevención primaria no están tan desarrolladas como la actitud terapeútica, pero es importante tener en cuenta mecanismos para identificar pacientes de alto riesgo. Entre ellos están los antecendentes de parto pretérmino, signos y síntomas, modificación cervical, etc. La rotura prematura de membranas y la corioamnionitis conllevan riesgo de parto pretérmino si se producen en edades gestaciones tempranas.

Palabras clave. Parto prematuro. Fibronectina. Modificación cervical. Rotura prematura de membranas. Corioamnionitis.

\begin{abstract}
The lower the gestational age at birth, the greater the risk of perinatal morbidity and mortality and maternal morbidity (increase in the number of caesareans, postnatal metritis). It is important for there to be a timely diagnosis of threatened preterm labour (TPL) in order to begin tocolytic treatment and to obtain efficient foetal pulmonary maturity. It is also necessary to distinguish between a true and a false TPL. There is a high tendency of overdiagnosis and overtreatment and prolonged hospitalisation is frequent. With cervical alteration and uterine dynamics tests it is possible to establish an accurate diagnosis that will result in suitable management. Early identification and primary prevention are not as developed as the therapeutic attitude, but it is important to bear in mind mechanisms for identifying high risk patients. They include antecedents of preterm labour, signs and symptoms, cervical alteration, etc. Premature rupture of membranes and chorioamnionitis involve the risk of preterm labour if they occur in early gestational ages.
\end{abstract}

Key words. Preterm labour. Fibronectin. Cervical alteration. Premature rupture of membranes. Chorioamnionitis.
Servicio de Obstetricia y Ginecología. Hospital Virgen del Camino. Pamplona.

\author{
Correspondencia: \\ Anabel Ochoa Prat \\ Servicio de Obstetricia y Ginecología \\ Hospital Virgen del Camino \\ Irunlarrea, 4 \\ 31008 Pamplona \\ E-mail: anabelochoap@hotmail.com
}




\section{INTRODUCCIÓN}

La amenaza de parto pretérmino (APP) se define como la presencia de contracciones con un ritmo de 4 cada 20 minutos o de 8 en 60 minutos entre la 22 y 37 semanas de gestación. Este hecho se debe asociar al menos a una de las siguientes circunstancias: modificación progresiva del cérvix y dilatación cervical $\geq$ a $2 \mathrm{~cm}$ y borramiento $\geq 80 \%$.

La prevalencia del parto pretérmino es del $7-10 \%$. Supone un $65 \%$ de muertes perinatales y la principal causa de morbilidad neonatal.

El 80\% de las consultas por APP no terminarán en un parto prematuro. Dos tercios de las APP no parirán en las siguientes 48 horas, y más de un tercio llegarán a término.

\section{CAUSAS Y FACTORES DE RIESGO}

No es una entidad clínica única. Confluyen una serie de etiologías y factores de riesgo diferentes que ponen en marcha el mecanismo de inicio del parto. Existen causas maternas como una enfermedad sistérmica grave, preeclampsia; causas uterinas; causas placentarias como placenta previa; causas del líquido amniótico como corioamnionitis, causas fetales como sufrimiento fetal agudo, etc ${ }^{1}$.

\section{EVALUACIÓN CLÍNICA}

Cuando existe la sospecha de que un cuadro clínico orienta hacia una APP se deben tener en cuenta diferentes parámetros:

- Historia clínica: contracciones, presión pélvica, dolor lumbar, hemorragia genital, aumento de flujo vaginal...

- Antecedentes personales y antecedentes obstétricos.

- Exploración general: pulso, tensión arterial y temperatura.

- Estimación de la edad gestacional, a partir de la fecha de la última regla o la fecha probable de parto calculada en la ecografía de primer trimestre.

- Exploración genital con la finalidad de realizar:
- Especulospia para ver las condiciones del cuello uterino, el estado de las membranas amnióticas y la presencia de hemorragia genital.

- Recogida de cultivo vagino-rectal para Estreptococo grupo B (EGB) y otros frotis y cultivos si fueran necesarios.

- Ecografía abdominal para visualizar número de fetos, estática fetal, estimar peso fetal y volumen de líquido amniótico y ver localización placentaria.

- Analítica básica: hemograma, coagulación, bioquímica y sedimento de orina.

- Valorar realizar urocultivo previo a tratamiento con antibióticos si se decide ingreso de la paciente.

\section{DIAGNÓSTICO}

- Valoración de la dinámica uterina mediante cardiotocografía externa o por palpación abdominal. No existe consenso sobre el número de contracciones necesarias para definir una APP pero generalmente se consideran:

- 4 en 20/30 minutos o bien 8 en 60 minutos.

- Duración de más de 30 segundos de cada contracción.

- Palpables y dolorosas.

Mediante la cardiotocografía externa también se valora el bienestar fetal. Las contracciones de Braxton-Hicks existen en un gran número de embarazos que finalizan a término la gestación. Es difícil diferenciarlas de las contracciones que van a producir modificaciones cervicales.

- Cambios cervicales comprobados mediante diferentes parámetros

\section{Tacto vaginal}

Se hace de forma previa para descartar anomalías de inserción placentaria y explorar vagina mediante especuloscopia por la posibilidad de encontrar la bolsa amniótica protuyendo a través de cérvix.

Se valora la dilatación, borramiento, posición, consistencia y altura de la presentación (Tabla 1). 
Tabla 1. Test de Bishop, valoración clínica del cuello.

\begin{tabular}{lcccc}
\hline & $\mathbf{0}$ & $\mathbf{1}$ & $\mathbf{2}$ & $\mathbf{3}$ \\
\hline Dilatación & 0 & $1-2 \mathrm{~cm}$ & $3-4 \mathrm{~cm}$ & $5-6 \mathrm{~cm}$ \\
\hline Borramiento & $0-30 \%$ & $40-50 \%$ & $60-70 \%$ & $\geq 80 \%$ \\
\hline Posición & Posterior & Media & Anterior & \\
\hline Consistencia & Dura & Media & Blanda & $+1 /+2$ \\
\hline Altura de la presenación & -3 & -2 & $-1 / 0$ & \\
\hline
\end{tabular}

Si el cérvix aparece borrado $>70 \%$ y/o dilatado de $2 \mathrm{~cm}$ o más, se considera diagnóstico de APP al igual que un borramiento y dilatación cervical progresivas ${ }^{2}$.

La realización del tacto vaginal es subjetiva y se puede perder mucha información. Hay una tasa de falsos positivos del $40 \%^{3}$.

\section{Marcador ecográfico}

La valoración del cuello uterino mediante la ecografía transvaginal es un método más objetivo, reproductible y con un valor más predictivo.

Para tener una correcta reproductibilidad de la ecografía transvaginal es necesario:
- Magnificar la imagen del cérvix al 75\% de la pantalla.

- El orificio cervical interno debe verse triangular o plano.

- Visualización del canal cervical en toda su longitud.

- El canal cervical debe estar equidistante del labio anterior y posterior.

- Realizar la ecografía con la vejiga vacía.

- No hacer excesiva presión sobre el cérvix o fondo de saco.

- Practicar tres mediciones y tener en cuenta la menor de ellas.

- Medir el canal cervical residual. No medir el funnel pero tenerlo en cuenta así como la dilatación del canal (Fig. 1).

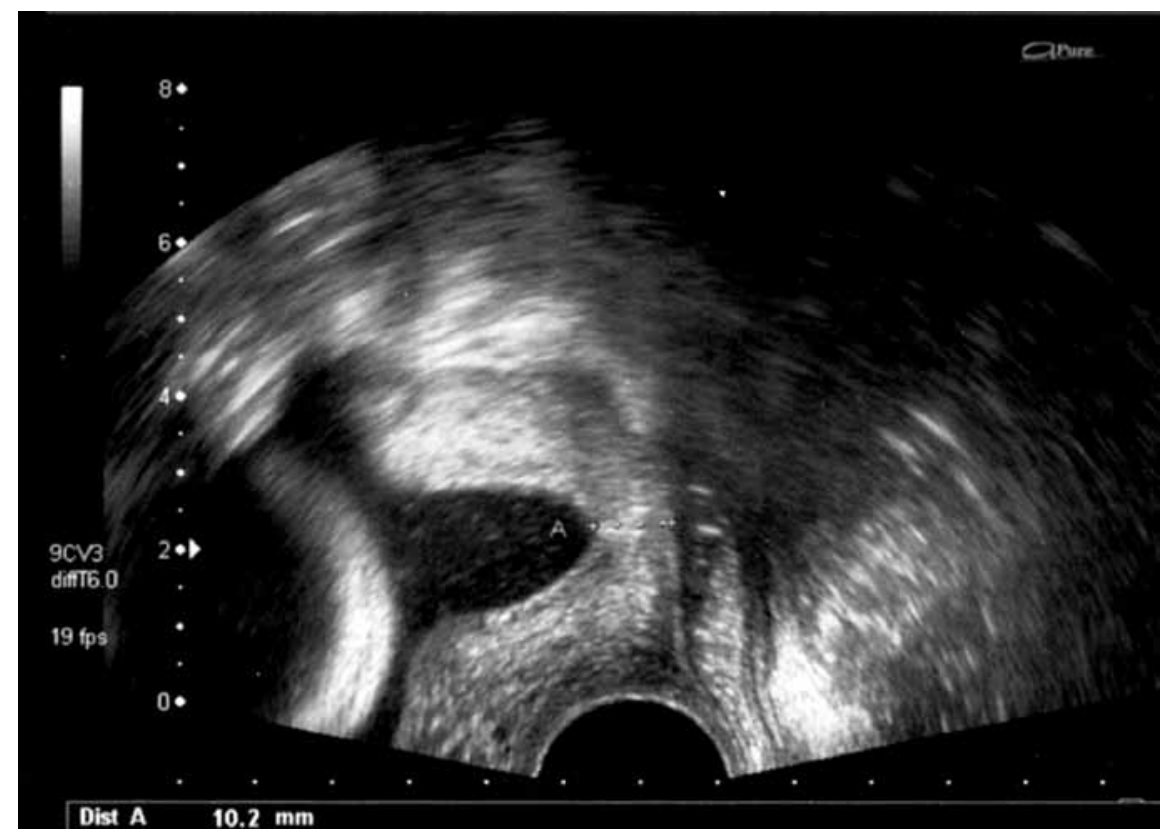

Figura 1. Ecografía de cérvix. Funnel. 
El borramiento cervical evoluciona en dirección cráneo-caudal. Hasta que los orificios cervicales interno y externo se unen,
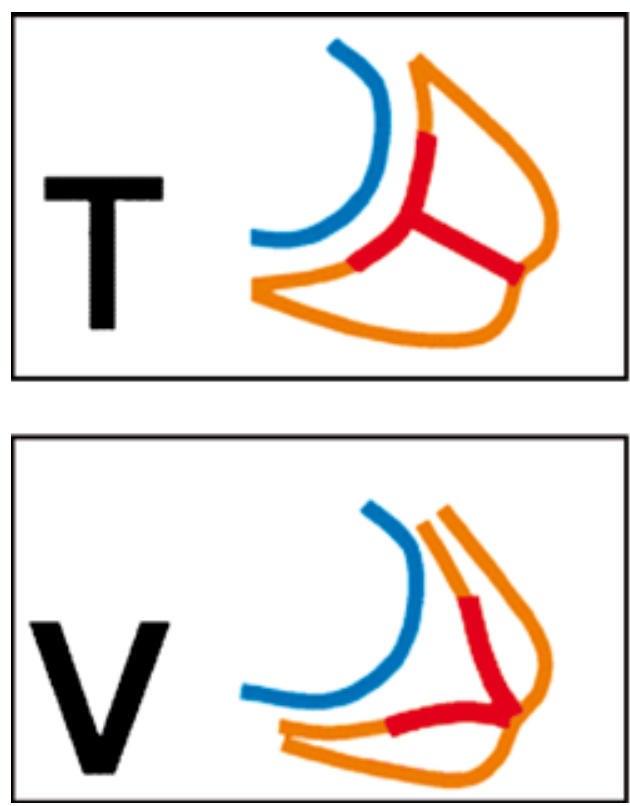

Figura 2. Modificaciones del cérvix en la APP.

Una medición $>30 \mathrm{~mm}$ tiene un elevado valor predictivo negativo para parto prematuro en mujeres sintomáticas. Excluye el diagnóstico de parto pretérmino ${ }^{4-6}$. El punto de corte para discriminar la verdadera de la falsa APP es $25 \mathrm{~mm}$. Una longitud cervical $<15 \mathrm{~mm}$ indica alta probabilidad de parto en 48 horas, 7 y 14 días. Un cérvix de $20-25 \mathrm{~mm}$ no siempre implica un parto prematuro, pero no puede excluirse ${ }^{4}$. el cuello se va acortando y el canal cervical adopta diferentes formas. (Figs. 2 y 3 ).
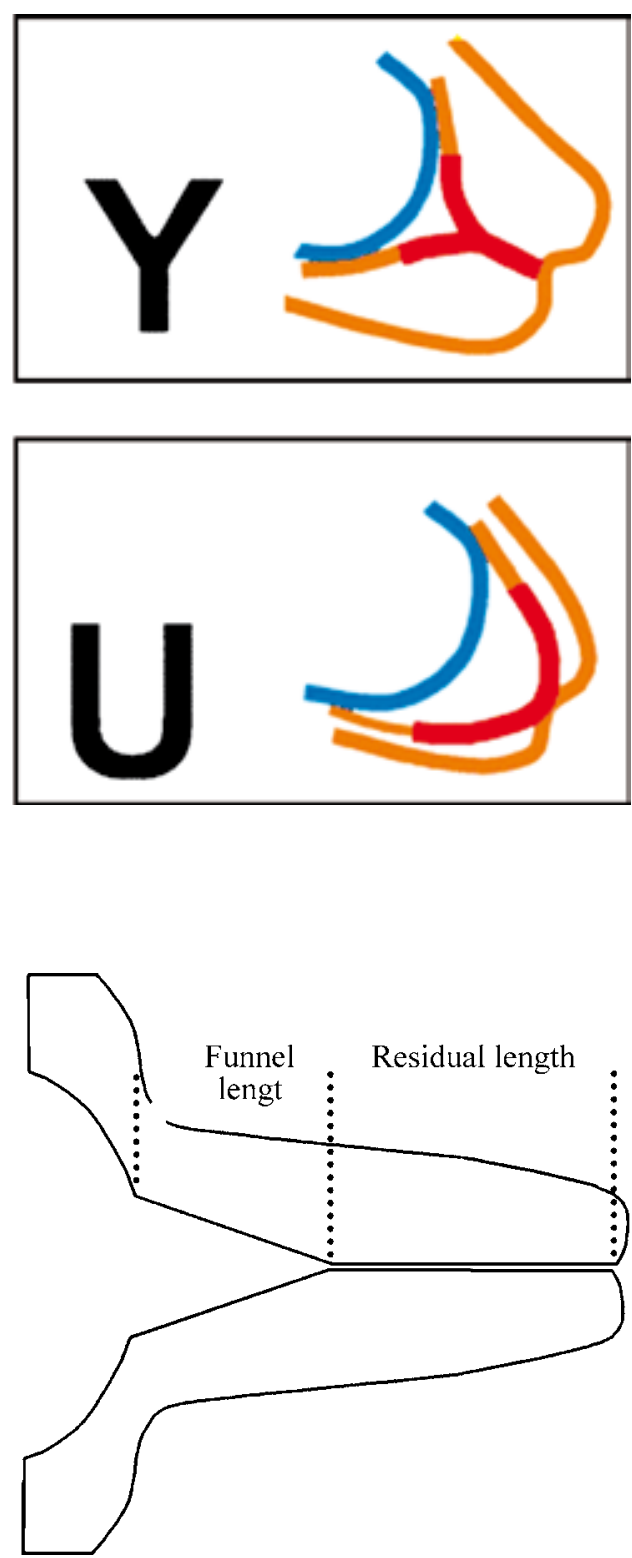

Figura 3. Funnel. 


\section{Marcador bioquímico}

La fibronectina fetal es una glucoproteína producida por el corion. Está relacionada con la adherencia de la placenta y membranas a la decidua. La separación entre decidua y corion conlleva un aumento de esta glucoproteína en secreción cérvicovaginal y esto reflejaría un comienzo espontáneo de parto.

Es normal detectar la fibronectina en gestaciones menores de 20 semanas y mayores de 37 semanas. Está contraindicada en los siguientes casos:

- Rotura prematura de membranas o $>3$ cm de dilatación

- Cerclaje

- Placenta previa y/o sangrado

- Exploraciones o relaciones en $<24$ horas

Está indicada en ${ }^{4}$ :

- Mujeres sintomáticas entre 24-34 semanas

- Dilatación $<3 \mathrm{~cm}$

- Membranas íntegras

Las mujeres con síntomas y signos de APP y un test de fibronectina negativo $(<50$ $\mathrm{ng} / \mathrm{ml}$ ) tienen un riesgo de parto prematuro en las dos semanas siguientes menor al $1 \%$. El valor predictivo negativo de esta prueba es muy elevado. Sirve más para descartar una APP que para confirmarla ${ }^{4}$.

Un test de fibronectina positivo tiene menos valor que una longitud de cérvix corta. El $30 \%$ de las pacientes con test positivo tendrá un parto pretérmino. La combinación de ambas pruebas para valorar el riesgo es mayor cuando la longitud cervical es $<30 \mathrm{~mm}$. Si la longitud de cérvix es $<20 \mathrm{~mm}$ ya se considera una APP y no sería necesario realizar la prueba de fibronectina ${ }^{6}$.

\section{Conducta a seguir ${ }^{7-8}$}

Tras la evaluación clínica inicial ${ }^{7-8}$ y los parámetros indicados para el diagnóstico de APP se pueden diferenciar diferentes situaciones (Figs. 4-6):

- Ausencia de contracciones y de modificaciones cervicales. En este caso se dará el alta hospitalaria sin ningún tratamiento.
- Fase prodrómica. Se caracteriza por: contracciones uterinas con frecuencia de 4 en 20/30 minutos o superior y/o cérvix borrado en menos de $70 \%$ con dilatación cervical inferior a $2 \mathrm{~cm}$.

- Conducta inicial: reposo, hidratación, evaluación del estado materno y del estado fetal.

- Revalorar tras 60-120 minutos con nueva exploración genital. Pueden ocurrir tres hechos:

1. Adinamia. Se descartaría una APP y se daría el alta a la paciente.

2. Exploración cervical similar y dinámica. En este caso se ingresará a la paciente manteniendo reposo durante 48 horas, evitando nuevas exploraciones. Si la paciente tiene factores de alto riesgo de parto pretérmino (gestación múltiple, antecedente de parto prematuro...) es recomendable la maduración pulmonar fetal mediante corticoides. Si tras 48 horas:

- no existe modificación cervical y desaparece la dinámica uterina se dará el alta hospitalaria a la paciente y seguirá con el control de embarazo habitual.

- persisten los signos y síntomas, sin haber cambios cervicales, la paciente permanecerá en reposo 48 horas nuevamente.

- existe modificación cervical se tratará de una APP en fase activa.

3. Modificación cervical. Se considerará una APP en fase activa.

- En la fase activa es necesario valorar:

- Contracciones uterinas con frecuencia de 4 en 20/30 minutos o superior.

- Cérvix borrado en más de 70\%, dilatación cervical igual o superior a $2 \mathrm{~cm}$.

- Modificaciones cervicales progresivas.

La conducta en este caso se basa en reposo, tratamiento tocolítico y maduración pulmonar fetal con corticoides. Es necesario ponerse en contacto con el equipo de neonatología por si el tratamiento fuera ineficaz y el parto se produjera en las próximas horas. Respecto a la vía de parto se 
optará por la vía vaginal, excepto cuando la presentación sea de nalgas y el peso estimado fetal menor de 1.500 gramos y/o la edad gestacional menor de 32 semanas. En estos casos se realizará una cesárea electiva.

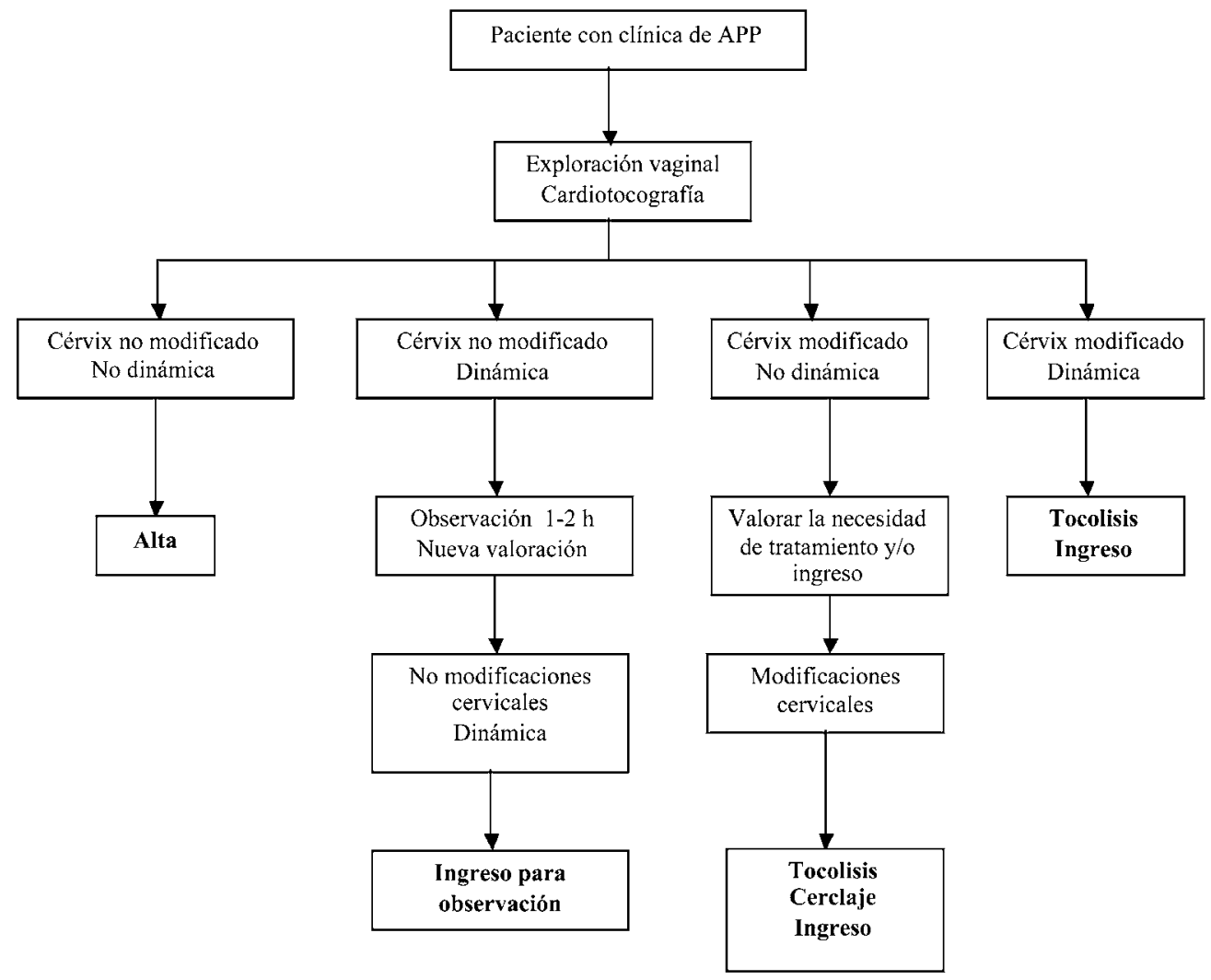

Figura 4. Conducta ante una gestante con clínica de $\mathrm{APP}^{7-8}$.

Si con el tratamiento tocolítico desaparece la dinámica uterina y el cérvix no se modifica, se mantiene durante 48 horas y se interrumpe. Si no reaparecen las contracciones el control de la gestación se hará de manera ambulatoria. Si reaparece la APP se iniciará el protocolo terapéutico.

Si hay ausencia de actividad uterina, pero el cuello uterino está modificado, podría tratarse de una incompetencia cervical. En este caso cabe la posibilidad de realizar un cerclaje de emergencia. La eficacia del cerclaje en mujeres con un cuello uterino acortado no ha sido claramente demostrada en estudios randomizados ${ }^{9-10}$. En algún estudio observacional se ha podido comprobar que el grupo de mujeres con cerclaje tiene significativamente menos partos pretérmino por debajo de semana 32 que las mujeres con cuello uterino acortado y conducta expectante ${ }^{11}$. Debe individualizarse cada caso y no está indicado en mujeres con edad gestacional viable ${ }^{9,12}$. Está contraindicado si existe rotura prematura de membranas, parto inminente, corioamnionitis y malformaciones fetales incompatibles con la vida. En estos momentos se están realizando estudios sobre la efectividad de la inserción de un pesario vaginal en pacientes con riesgo de parto espontáneo pretérmino ${ }^{13}$. 


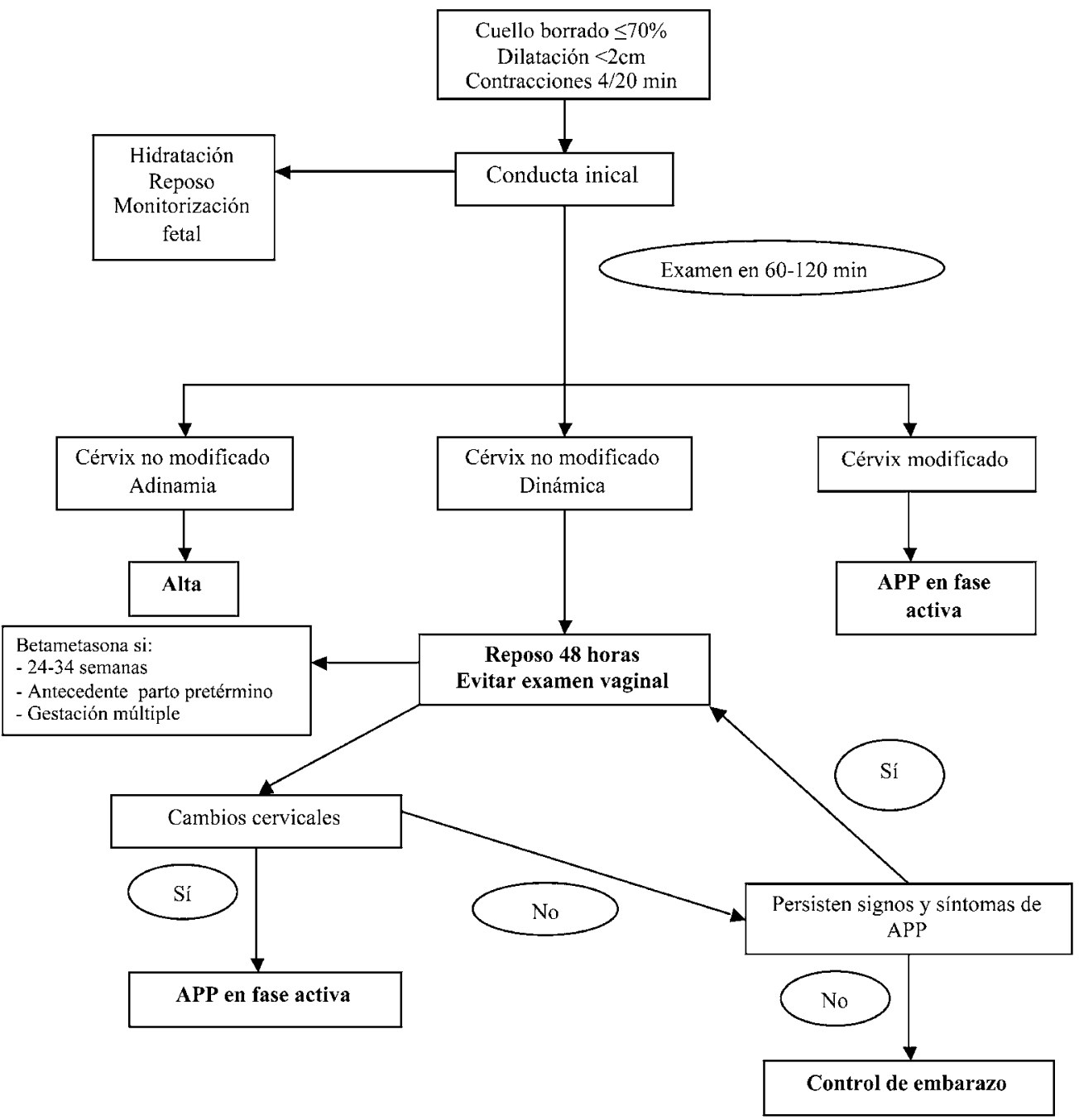

Figura 5. Conducta ante una APP en fase prodrómica ${ }^{7-8}$. 


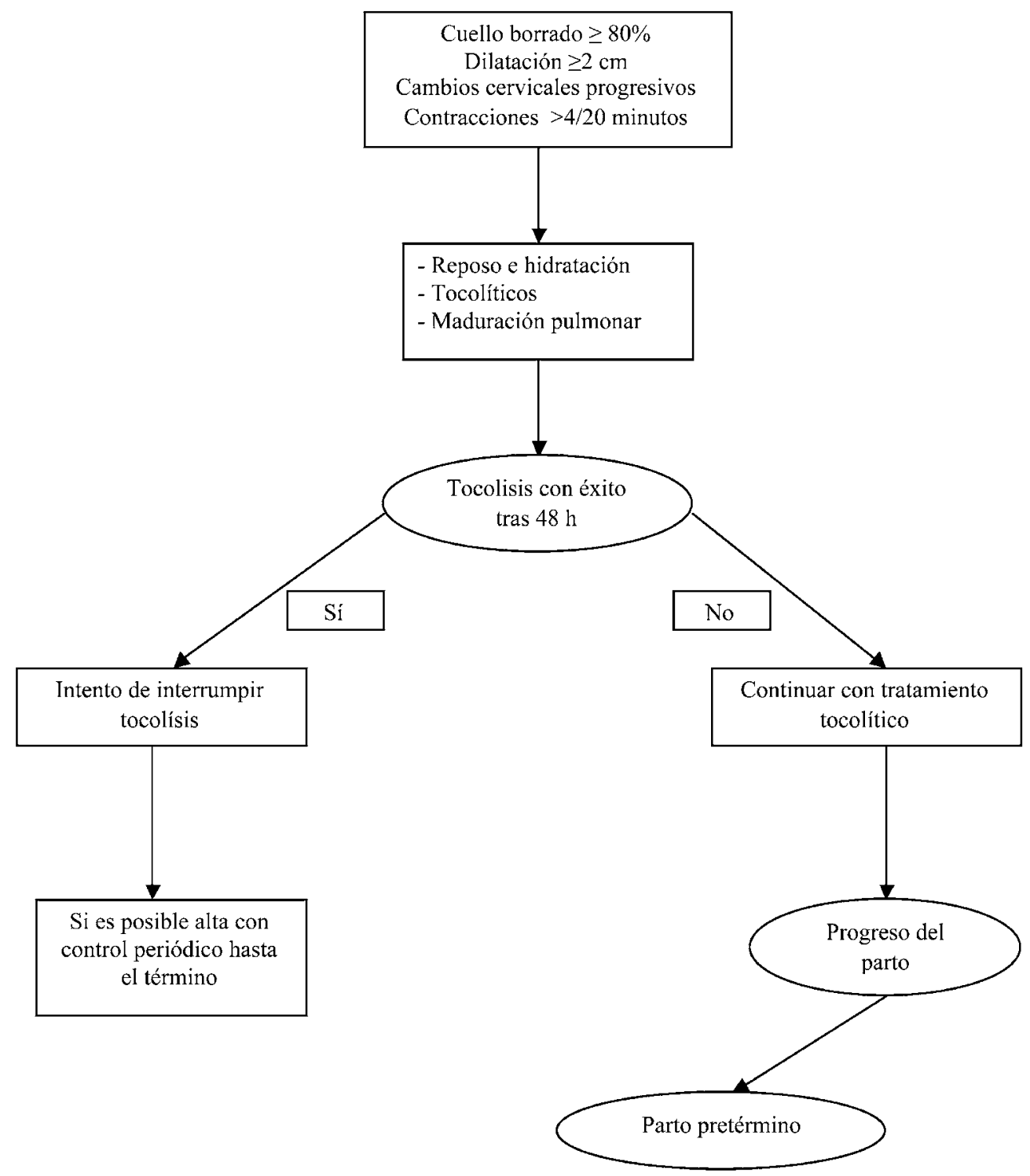

Figura 6. Conducta ante una APP en fase activa ${ }^{7-8}$.

\section{TRATAMIENTO}

1. Tratamiento etiológico cuando se conozca la causa de la APP (infección vaginal o urinaria, corioamnionitis, rotura prematura de membranas...)

2. Reposo e hidratación. Su eficacia real parece dudosa y su uso rutinario, in- cluso para la diferenciación de la verdadera amenaza de parto prematuro no se recomienda. El éxito de estas medidas puede ser debido a que son mujeres con falsas APP que se resolverían sin tratamiento.

3. Tratamiento tocolítico ${ }^{8}$ (Tabla 2). 
Tabla 2. Fármacos tocolíticos ${ }^{14}$.

\begin{tabular}{|c|c|c|c|c|c|}
\hline $\begin{array}{c}\text { Agente } \\
\text { tocolítico }\end{array}$ & $\begin{array}{c}\text { Dosis y } \\
\text { administración }\end{array}$ & Contraindicaciones & Efectos secundarios & Vigilar & Comentarios \\
\hline $\begin{array}{l}\text { Bmimético } \\
\text { Ritodrine * }\end{array}$ & $\begin{array}{l}\text { - } 4 \text { ampollas en } \\
500 \text { ml de suero } \\
\text { glucosado al } 5 \% . \\
\text { - Dosis inicial de } 12 \\
\text { ml/hora, aumento } 12 \\
\text { ml/hora cada 10-15 } \\
\text { min hasta cese de } \\
\text { dinámica o efectos } \\
\text { secundarios } \\
\text { - Dosis máxima = } \\
54 \text { ml/hora } \\
\text { - Reducir la infusión } \\
\text { con cese de } \\
\text { dinámica (a los } 60 \\
\text { min) y mantener } 12 \\
\text { horas mínimo }\end{array}$ & $\begin{array}{l}\text { - Arritmia cardiaca } \\
\text { - Uso digital } \\
\text { - Enfermedad } \\
\text { cardiaca restrictiva } \\
\text { - Hipertiroidismo } \\
\text { - Diabetes mellitus } \\
\text { mal controlada } \\
\text { - Anemia severa }\end{array}$ & $\begin{array}{l}\text { - Arritmia cardiaca, } \\
\text { edema pulmonar, } \\
\text { isquemia } \\
\text { miocárdica, } \\
\text { hipotensión, } \\
\text { taquicardia } \\
\text { - Hiperglucemia, } \\
\text { hipocaliemia, } \\
\text { antidiuresis, } \\
\text { alteraciones } \\
\text { tiroideas } \\
\text { - Temblor, } \\
\text { palpitaciones, } \\
\text { nerviosismo, } \\
\text { náuseas y vómitos, } \\
\text { fiebre y cefalea. } \\
\text { - Perinatales: } \\
\text { taquicardia, } \\
\text { hipoglucemia, } \\
\text { hiperbilirrubinemia, } \\
\text { hicocalcemia, } \\
\text { hemorragia } \\
\text { intramentricular } \\
\text { neonatal. }\end{array}$ & $\begin{array}{l}\text { - Balance hídrico } \\
\text { - Pulso (<140 pm) } \\
\text { - Tensión arterial } \\
\text { - Frecuencia } \\
\text { respiratoria } \\
\text { - Auscultación } \\
\text { pulmonar } \\
\text { - Electrolitos (K) } \\
\text { - Glucosa }\end{array}$ & $\begin{array}{l}\text { Su uso como } \\
\text { tocolítico de elección } \\
\text { ha disminuido a favor } \\
\text { de otros fármacos. } \\
\text { Por vía oral no tiene } \\
\text { eficacia } \\
\text { (ni de entrada } \\
\text { ni como } \\
\text { mantenimiento) } \\
\text { *Aprobado en España } \\
\text { para APP }\end{array}$ \\
\hline $\begin{array}{l}\text { Sulfato de } \\
\text { Magnesio }\end{array}$ & $\begin{array}{l}\text { 4-6 g en bolo en } 20 \\
\text { min, luego } 2-3 \mathrm{~g} / \\
\text { hora en infusión } \\
\text { mantenimiento } \\
\text { - Reducir la infusión } \\
\text { al máximo con } \\
\text { cese de dinámica } \\
\text { y mantener 12-24 } \\
\text { horas } \\
\text { - Niveles terapéuticos } \\
\text { para tocolisis 5-8 } \\
\text { mg/dl } \\
\text { - Si intoxicación debe } \\
\text { usarse gluconato } \\
\text { de calcio, } 1 \mathrm{~g} \\
\text { intravenoso }\end{array}$ & $\begin{array}{l}\text { - Miastenia gravis } \\
\text { - Bloqueo cardiaco } \\
\text { - Infarto reciente } \\
\text { - Alteración renal }\end{array}$ & $\begin{array}{l}\text { - Enrojecimiento } \\
\text { facial, calores, } \\
\text { sofocación, letargo, } \\
\text { cefalea, náuseas } \\
\text { - Debilidad muscular, } \\
\text { diplopia, sequedad } \\
\text { bucal } \\
\text { - Edema pulmonar, } \\
\text { fallo cardiaco } \\
\text { - Perinatales: } \\
\text { depresión del } \\
\text { sistema nervioso } \\
\text { central, hipotonía } \\
\text { profunda, } \\
\text { disminución de } \\
\text { frecuencia cardiaca } \\
\text { fetal, anormalidades } \\
\text { esqueléticas }\end{array}$ & $\begin{array}{l}\text { - Frecuencia } \\
\text { respiratoria } \\
\text { - Reflejos tendinosos } \\
\text { - Magnesemia cada } \\
\text { 2-4 horas }\end{array}$ & $\begin{array}{l}\text { Los beneficios no } \\
\text { compensan los } \\
\text { efectos secundarios. } \\
\text { NO asociar a } \\
\text { bloqueantes del } \\
\text { calcio } \\
\text { No ha sido } \\
\text { demostrada su } \\
\text { eficacia como } \\
\text { tocolítico }\end{array}$ \\
\hline $\begin{array}{l}\text { Bloqueantes del } \\
\text { calcio } \\
\text { Nifedipino }\end{array}$ & $\begin{array}{l}\text { - } 10 \mathrm{mg} / 20 \mathrm{~min} \text { vía } \\
\text { oral dosis ataque } \\
\text { (4 dosis máximo), } \\
\text { luego } 10-20 \mathrm{mg} \text { cada } \\
\text { 4-6 horas } \\
\text { - Máximo } 120 \mathrm{mg} / 24 \\
\text { horas }\end{array}$ & $\begin{array}{l}\text { - Enfermedad } \\
\text { cardiaca (estenosis } \\
\text { ao), enfermedad } \\
\text { renal severa, } \\
\text { hipotensión } \\
\text { materna }(<90 / 50)\end{array}$ & $\begin{array}{l}\text { - Enrojecimiento } \\
\text { facial, cefalea, } \\
\text { vértigo, náuseas, } \\
\text { hipotensión } \\
\text { transitoria } \\
\text { - Perinatales: no hay } \\
\text { evidencia }\end{array}$ & - Tensión arterial & $\begin{array}{l}\text { Superior a los } \\
\text { B miméticos en } \\
\text { meta-análisis. } \\
\text { No hay estudios } \\
\text { randomizados } \\
\text { comparándolo } \\
\text { con placebo o sin } \\
\text { tratamiento }\end{array}$ \\
\hline
\end{tabular}




\begin{tabular}{|c|c|c|c|c|c|}
\hline $\begin{array}{c}\text { Agente } \\
\text { tocolítico }\end{array}$ & $\begin{array}{c}\text { Dosis y } \\
\text { administración }\end{array}$ & Contraindicaciones & Efectos secundarios & Vigilar & Comentarios \\
\hline $\begin{array}{l}\text { Inhibidores } \\
\text { de las } \\
\text { Prostaglandinas } \\
\text { Indometacina }\end{array}$ & $\begin{array}{l}\text { - Dosis ataque 50-100 } \\
\text { mg vía oral ó rectal, } \\
\text { luego } 25-50 \mathrm{mg} / 4-6 \\
\text { horas vía oral de } \\
\text { mantenimiento } \\
\text { - Máximo } 200 \mathrm{mg} / 24 \\
\text { horas } \\
\text { - Vía vaginal: } 100 \\
\text { mg/12 horas. Es tan } \\
\text { eficaz o más }\end{array}$ & $\begin{array}{l}\text { - Enfermedad renal } 0 \\
\text { hepática graves } \\
\text { - Ulcus gástrico } \\
\text { - Asma inducido por } \\
\text { fármacos } \\
\text { - Alteraciones } \\
\text { coagulación o } \\
\text { trombopenia } \\
\text { - Alergia a aspirina }\end{array}$ & $\begin{array}{l}\text { - Nausea, pirosis, } \\
\text { hemorragia } \\
\text { digestiva } \\
\text { - Perinatales: } \\
\text { oligohidramnios, } \\
\text { cierre del ductus } \\
\text { arterioso, } \\
\text { hipertensión } \\
\text { pulmonar, } \\
\text { hemorragia } \\
\text { intraventricular, } \\
\text { hiperbilirrubinemia, } \\
\text { enterocolitis } \\
\text { necrotizante }\end{array}$ & \begin{tabular}{|l} 
- Índice de líquido \\
amniótico diario si \\
terapia de $>48$ horas \\
(suspender $\mathrm{si}<5$ ) \\
- Índice de \\
pulsatilidad del \\
ductus (suspender \\
si $<2 \mathrm{~cm} / \mathrm{seg}$ )
\end{tabular} & $\begin{array}{l}\text { Su eficacia está } \\
\text { demostrada en } \\
\text { estudios control } \\
\text { randomizados y } \\
\text { prospectivos. De } \\
\text { primera elección en } \\
\text { múltiples escenarios } \\
\text { por su eficacia, uso } \\
\text { vía oral y escasos } \\
\text { efectos maternos. } \\
\text { También de elección } \\
\text { como } 2^{0} \text { agente } \\
\text { combinado si fracasa } \\
\text { la monoterapia. } \\
\text { No usar en > } 32 \\
\text { semanas }\end{array}$ \\
\hline $\begin{array}{l}\text { Antagonistas de } \\
\text { la oxitocina } \\
\text { Atosibán * }\end{array}$ & $\begin{array}{l}\text { - Bolo IV de } 0,9 \mathrm{ml} \\
\text { - } 24 \mathrm{ml} / \mathrm{h} \text { durante } 3 \\
\text { horas } \\
\text { - } 8 \mathrm{ml} / \mathrm{h} \text { durante } 3,5 \\
\text { horas } \\
\text { - } 8 \mathrm{ml} / \mathrm{h} \text { hasta } 45 \\
\text { horas. } \\
\text { Se pueden repetir } \\
\text { varios ciclos. }\end{array}$ & $\begin{array}{l}\text { - Hipersensibilidad al } \\
\text { producto }\end{array}$ & $\begin{array}{l}\text { - Náuseas, } \\
\text { vómitos, cefalea, } \\
\text { dolor torácico, } \\
\text { taquicardia }\end{array}$ & No precisa & $\begin{array}{l}\text { Eficacia comparable } \\
\text { a Ritodrine con } \\
\text { efectos secundarios } \\
\text { mínimos. } \\
\text { Mínimo impacto } \\
\text { sobre resultados } \\
\text { perinatales. } \\
\text { * Aprobado en } \\
\text { España para APP }\end{array}$ \\
\hline $\begin{array}{l}\text { Inhibidores } \\
\text { de la ciclo- } \\
\text { oxigenasa } \\
\text { Sulindac }\end{array}$ & $\begin{array}{l}\text { - } 200 \text { mg vía oral/12 } \\
\text { horas durante } 48 \\
\text { horas }\end{array}$ & $\begin{array}{l}\text { - Las mismas } \\
\text { que para la } \\
\text { indometacina }\end{array}$ & $\begin{array}{l}\text { - Alteración de } \\
\text { la coagulación, } \\
\text { trastornos } \\
\text { gastrointestinales } \\
\text { - Perinatales: } \\
\text { Contracción del } \\
\text { ductus arterioso } \\
\text { y disminución del } \\
\text { líquido amniótico }\end{array}$ & $\begin{array}{l}\text { - Igual que con la } \\
\text { indometacina }\end{array}$ & $\begin{array}{l}\text { La COX-2 está } \\
\text { regulada en el } \\
\text { segmento uterino, } \\
\text { membranas } \\
\text { ovulares y cérvix. } \\
\text { Su inhibición } \\
\text { selectiva puede } \\
\text { ser más efectiva } \\
\text { que la inhibición } \\
\text { no selectiva por la } \\
\text { indometacina } \\
\end{array}$ \\
\hline
\end{tabular}

La decisión de tratar una APP de edad gestacional entre 22-24 semanas, se debe tomar conjuntamente con los padres tras informarles de los resultados neonatales y de las secuelas esperadas en cada caso, así como de las posibilidades de éxito.

No disminuye la frecuencia de parto pretérmino. Su principal objetivo es prolongar la gestación al menos 24-48 horas para permitir la maduración pulmonar fetal con corticoides.

Contraindicaciones:

- Corioamnionitis.

- Hemorragia materna grave.
- Preeclampsia severa o eclampsia.

- Trabajo de parto avanzado.

- Muerte fetal intraútero.

- Malformación fetal incompatible con la vida.

- Retraso de crecimiento intrauterino severo.

- Sospecha de pérdida de bienestar fetal.

La elección del fármaco tocolítico debe hacerse en función de los efectos secundarios. La eficacia útero-inhibidora es parecida en todos los fármacos. El tratamiento tocolítico de mantenimiento no ha mostrado ningún beneficio. 


\section{Corticoides ${ }^{14}$}

Se debe administrar en gestantes de entre 24-34 semanas, con riesgo de parto pretérmino que no precisen extracción fetal urgente. El efecto máximo se consigue entre las 24 horas y los 7 días después de haber comenzado la administración. El tratamiento de menos de 24 horas también tiene efectos beneficiosos, ya que se asocia con una disminución de la mortalidad neonatal, distrés respiratorio y hemorragia intraventricular.

No existe evidencia para recomendar dosis repetidas de corticoides semanales en mujeres en las que persiste el riesgo de parto prematuro.

Están contraindicados si existe corioamnionitis, feto muerto, malformación incompatible con la vida, pérdida de bienestar fetal, desprendimiento de placenta, tuberculosis o porfiria. La asociación de corticoides con betamiméticos en gestaciones múltiples conlleva un riesgo aumentado de edema agudo de pulmón. En las gestantes diabéticas se incrementa el riesgo de descompensación metabólica.

Pauta de administración:

- Betametasona: $12 \mathrm{mg} / 24$ horas, 2 dosis i.m.; la $2^{\text {a }}$ dosis puede administrarse a las 12 horas si se prevé que se va a desencadenar el parto. Es el corticoide de elección.

- Dexametasona: $6 \mathrm{mg} / 12$ horas, 4 dosis i.m.

\section{Tratamiento antibiótico.}

$\mathrm{Si}$ existe rotura prematura de membranas, vaginosis bacteriana o colonización por EGB se debe utilizar tratamiento antibiótico específico. El uso rutinario de antibióticos en las APP no es eficaz.

\section{ROTURA PREMATURA DE MEMBRANAS}

La rotura prematura de membranas (RPM) consiste en la pérdida de integridad de las membranas ovulares antes del inicio del parto, con la consiguiente salida de líquido amniótico y la puesta en comunicación de la cavidad amniótica con el canal endocervical y la vagina. La prevalencia en gestaciones menores de 37 semanas es del 1-4\%.

La causa de la RPM es multifactorial al igual que la APP: tabaquismo, déficit de vi- tamina C, sobredistensión uterina, hemorragia preparto... Pero sobre todo destacan:

- Parto pretérmino previo y/o antecedente de RPM.

- Lesiones mecánicas como la amniocentesis genética, fetoscopia y cerclaje cervical.

- Infección del tracto genital o intraamniótica.

Según la localización la RPM se clasifica en:

- Rotura alta (persiste bolsa amniótica por delante de la presentación)

- Rotura baja (no hay bolsa amniótica por delante de la presentación)

En ambas ocasiones se actúa de la misma manera, no hay diferencias de cara al tratamiento porque no se ha demostrado menor riesgo de infección en la rotura alta.

Según la edad gestacional se clasifica en $\leq 24$ semanas, $24-34$ semanas $\mathrm{y} \geq 35$ semanas (esta clasificación es orientativa y depende de cada centro y de los resultados perinatales).

\section{Diagnóstico}

\section{Anamnesis}

Es importante descartar otros diagnósticos como son la incontinencia de orina o el aumento de flujo vaginal, los cuales presentan una clínica similar y pueden condicionar actitudes erróneas.

\section{Exploración física ${ }^{8-14}$}

- Genitales externos: puede observarse salida de líquido amniótico a través de vagina, espontáneamente o con maniobra de Valsalva.

- Espéculo: para visualizar la salida de líquido amniótico a través del orifico cervical externo u objetivarlo en fondo de saco vaginal posterior. Las maniobras de Valsalva también pueden ser útiles en este caso. Otra opción es dejar un tiempo a la paciente semisentada con una compresa seca y revalorarla posteriormente. Se deben evitar los tactos vaginales. Sólo se harán si es necesario valorar el índice de Bishop, sin introducir los dedos en el canal cervical. 


\section{Pruebas complementarias}

- Determinación del pH del fondo de saco vaginal posterior. El $\mathrm{pH}$ vaginal en condiciones normales es ácido (4,5-5). El líquido amniótico tiene un pH básico $(7-7,5)$. La presencia de líquido amniótico en vagina aumentará el $\mathrm{pH}$, pudiendo alcanzar un valor $>$ de 6,4 . En este caso el papel de nitracina vira a azul. Los falsos positivos pueden darse con la presencia de semen, sangre, vaginosis bacteriana, moco cervical o antisépticos.

- Cristalización en hojas de helecho del líquido amniótico. Al observar con microscopio óptico el líquido amniótico seco en un portaobjetos se ven formaciones en hojas de helecho. Darán falsos positivos la presencia de semen, sangre, moco cervical $o$ antisépticos.

- PROM test. Permite la detección de IGFBP-1 (insulin-like growth factor binding protein-1). Esta sustancia está presente en líquido amniótico, no en vagina. Durante el examen vaginal con espéculo se toma la muestra con un hisopo del fondo de saco vaginal posterior durante 10-15 segundos. Puede existir un falso negativo si ha cesado la salida de líquido amniótico 12 horas antes de tomar la muestra. La presencia de sangre puede dar un falso positivo. No ocurre así con orina o semen.

- Test de fluoresceína. Consiste en la instilación intraamniótica de fluoresceína mediante amniocentesis. Se coloca una compresa en genitales externos y se observa la presencia de colorante en la misma con luz de Word tras 30 minutos. Este hecho indicaría una RPM. Esta prueba debe realizarse cuando se dude del diagnóstico y éste tenga implicaciones en la actitud obstétrica.

- Ecografía. Permite valorar el índice de líquido amniótico. La existencia de un oligoamnios no diagnosticado previamente podrá orientar hacia una RPM. Presenta falsos positivos (causas de oligoamnios diferentes a la RPM) y falsos negativos (RPM con cantidad de líquido amniótico normal).

\section{Evaluación clínica inicial}

- Confirmar la RPM con las pautas mencionadas anteriormente: anamnesis, exploración física y pruebas complementarias.

- Valoración del riesgo de inmadurez fetal. Es importante establecer con exactitud la edad gestacional.

- Las pacientes con feto viable y RPM deben hospitalizarse hasta el parto.

- Al ingreso es necesario hacer:

- Control de temperatura y pulso cada 6 horas y de tensión arterial cada 8 horas.

- Comprobar bienestar fetal y existencia de actividad uterina mediante cardiotocografía.

- Valorar el riesgo de infección mediante una analítica de sangre con hemograma con recuento de serie blanca y roja y determinación de proteína $C$ reactiva. Se realizarán tomas para cultivo vagino-rectal para EGB y urocultivo. Se pueden realizar también cultivos para chlamydias y gonococo del exudado endocervical.

- Valorar amniocentesis si existen dudas sobre la madurez pulmonar o infección amniótica.

- Si se evidencia corioamnionitis clínica, abruptio placentae o compromiso fetal se debe finalizar la gestación de manera inmediata.

\section{Tratamiento}

- Reposo en cama.

- Tratamiento antibiótico ${ }^{8}$. La profilaxis antibiótica ha demostrado retrasar el parto, disminuir el número de infecciones maternas, infecciones neonatales, distrés respiratorio, secuelas neurológicas mayores y la mortalidad antenatal y neonatal. Por todas estas razones se recomienda su uso profiláctico en el tratamiento expectante en la RPM pretérmino. Respecto al tipo de antibiótico a utilizar existen diferentes pautas propuestas y combinaciones:

- Ampicilina $2 \mathrm{~g}$ iv/6h + Eritromicina 250 $\mathrm{mg}$ iv/6h durante 48 horas, seguido de amoxicilina $500 \mathrm{mg}$ vo/8h + Eritromicina $250 \mathrm{mg}$ vo/6h durante 5 días. 
- Eritromicina $250 \mathrm{mg}$ vo/6h durante 10 días.

- Eritromicina $250 \mathrm{mg}$ iv/6h durante 24 horas, seguido de eritromicina 500 mg vo/6h hasta una semana.

- Ampicilina $1 \mathrm{~g}$ iv/6h durante 24 horas, seguido de amoxicilina $500 \mathrm{mg}$ vo/8h hasta una semana o hasta recibir el resultado negativo del cultivo vagino-rectal y de líquido amniótico.

La elección de alguna de estas pautas dependerá de la experiencia de cada centro, así como de la sensibilidad de los microorganismos prevalentes.

Si el cultivo de EGB es positivo el tratamiento antibiótico durante el manejo expectante debe durar al menos 7 días. La profilaxis antibiótica se debe repetir cuando se instaure el parto.
- Corticoides. Ver tratamiento con corticoides en APP.

- Tratamiento tocolítico. Si se evidencia dinámica uterina o modificaciones cervicales en gestaciones con inmadurez pulmonar se utilizarán fármacos tocolíticos durante 48 horas para permitir la administración de corticoides y antibióticos. No es conveniente utilizar indometacina debido a que puede enmascarar la fiebre.

\section{Conducta a seguir}

El protocolo que se presenta es el llevado a cabo en el Hospital Virgen del Camino (Fig. 7).

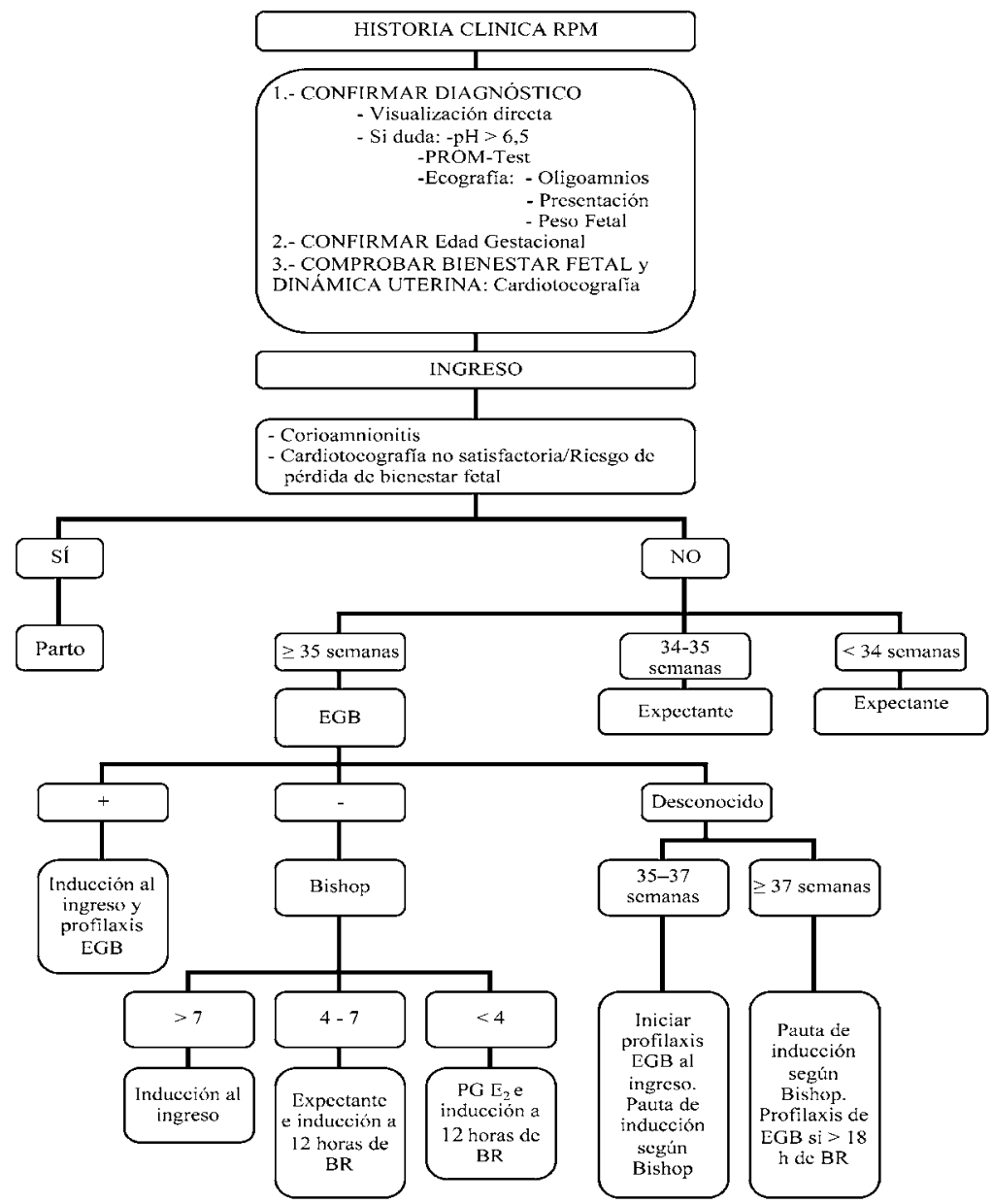

Figura 7. Conduta a seguir en la RPM. Protocolo de actuación del Hospital Virgen del Camino. 


\section{Gestación menor de 26 semanas (Gestación previable)}

La probabilidad de supervivencia fetal es reducida y en caso de sobrevivir, las secuelas son muy elevadas. Esta edad gestacional corresponde con la formación de la fase canalicular del desarrollo pulmonar. El oligoamnios es un factor de riesgo añadido para la hipoplasia pulmonar ${ }^{15}$. Hay que informar a los padres de estos datos $y$ hacerles partícipes de la decisión a tomar. Se debe individualizar en cada caso la decisión de finalizar la gestación o de intentar un tratamiento conservador con conducta expectante. Actualmente en algunos centros y según el equipo de neonatología el límite para actuar de manera expectante ante una RPM son las 24 semanas.

\section{Gestación entre 26 y 34 semanas}

Conducta expectante. Iniciar maduración pulmonar fetal con corticoides tras descartar corioamnionitis o compromiso fetal y comenzar con antibioterapia profiláctica. Si se evidencia una amenaza de parto prematuro se administrarán fármacos tocolíticos durante 48 horas. Si se desencadena el parto se efectuará profilaxis de la infección por EBG si el cultivo es positivo o desconocido $^{16}$. Si la RPM se produce en una gestante con cerclaje, se realizará la misma conducta retirando el cerclaje ante la sospecha de corioamnionitis o en semana 32 . Algunos autores recomiendan su retirada a partir de semana 28.

\section{Gestación entre 34 y 35 semanas}

Conducta expectante. Se administrará antibioterapia profiláctica. Si se aprecian signos de corioamnionitis o compromiso fetal se finalizará la gestación. Si se instaura dinámica uterina se dejará evolucionar el parto, teniendo en cuenta la profilaxis para EGB si el cultivo ha sido positivo o si es desconocido.

\section{Gestación $\geq 35$ semanas}

Si el cultivo para EGB es positivo se realizará inducción de parto con profilaxis antibiótica. Si el cultivo para EGB es negativo la pauta de actuación dependerá del Test de Bishop.

- Si es $>7$, se inducirá el parto al ingreso.

- Si es 4-7, se mantendrá una conducta expectante durante 12 horas de BR. Si no se ha desencadenado el parto en este tiempo se inducirá con oxitocina.

- Si es <4 se utilizarán prostaglandinas $\mathrm{E}_{2} \mathrm{y}$ tras 12 horas de BR se inducirá el parto con oxitocina.

Si el cultivo es desconocido y la edad gestacional está entre 35 y 37 semanas se iniciará profilaxis para EBG y se inducirá según el test de Bishop. Si la gestación es $\leq 37$ semanas se realizará una pauta de inducción según el test de Bishop comenzando con profilaxis para EGB tras 18 horas de BR.

\section{CORIOAMNIONITIS}

Se define como la presencia de gérmenes en el líquido amniótico (estéril en condiciones normales) que conllevará manifestaciones clínico-analíticas para la madre y/o para el feto. Es más frecuente cuando se asocia RPM en una gestación pretérmino.

\section{Diagnóstico}

- Clínico. Presencia de fiebre $\left(\geq 37,8^{\circ} \mathrm{C}\right)$ $\mathrm{y}$ al menos dos de los siguientes criterios $^{17}$ :

- Taquicardia materna $>100 \mathrm{lpm}$.

- Taquicardia fetal >160 lpm.

- Dolor uterino.

- Líquido amniótico maloliente, fétido.

- Leucocitosis materna > 15.000 leucocitos $/ \mathrm{mm}^{3}$, con desviación izquierda.

- Analítica (realizar de forma sistemática)

- Hemograma: leucocitos >15.000 y desviación a la izquierda.

- Proteína $\mathrm{C}$ reactiva elevada.

- Hemocultivo. El resultado tarda 48 horas y suele ser positivo en el $10 \%$ de las corioamnionitis.

- Comprobar bienestar fetal (realizar de forma sistemática).

- Cardiotocografía basal no estresante. Un patrón no reactivo junto a ta- 
quicardia fetal puede ser un signo precoz de infección intraamniótica.

- Ecografía. La ausencia de movimientos respiratorios y de forma más tardía con ausencia de movimientos fetales y de tono fetal se asocian a corioamnionitis.

- Amniocentesis (opcional).

Es un método invasivo que puede realizarse si existen dudas con la clínica y las exploraciones complementarias. En el líquido amniótico se pueden determinar:

- Recuento de leucocitos. Si >50 leucocitos $/ \mathrm{mm}^{3}$.

- Glucosa: <14 mg/dl.

- Tinción de Gram. Si es positiva es diagnóstica, pero si es negativa no excluye. No identifica el Micoplasma.

- Cultivo de líquido amniótico. El resultado tarda unas 48 horas.

\section{Tratamiento}

Consiste en tratamiento antibiótico y finalizar la gestación. La administración de antibióticos debe ser terapéutica y no profiláctica. Pautas:

- Ampicilina, $2 \mathrm{~g} / 6 \mathrm{~h}$ ev o penicilina, $5 \mathrm{mi}$ llones UI/6h ev + gentamicina, $1,5 \mathrm{mg} /$ $\mathrm{Kg} / 8 \mathrm{~h}$ ó $7 \mathrm{mg} / \mathrm{Kg}$ de peso ideal $/ 24 \mathrm{~h}$.

- Clindamicina, $900 \mathrm{mg} / 8 \mathrm{~h} \mathrm{ev}+$ gentamicina, $1,5 \mathrm{mg} / \mathrm{Kg} / 8 \mathrm{~h}$ ó $7 \mathrm{mg} / \mathrm{Kg}$ de peso ideal/24 h en alérgicos a beta-lactámicos.

- Si se realiza parto por cesárea es conveniente añadir postoperatoriamente clindamicina, $900 / 8 \mathrm{~h}$ ev o metronidazol, $500 \mathrm{mg} / 8-12 \mathrm{~h}$ para cubrir anaerobios.

Los niveles séricos de ampicilina y gentamicina son máximos a las 2-6 h de su administración.

\section{BIBLIOGRAFÍA}

1. IAMS JD. Preterm birth. En: Gabbe SG, Niebyl JR, SimpsonJL, eds. Obstetrics: Normal and problem pregnancies. 4thed. Philadelphia: Churchill and Livingstone, 2002: 755-826.

2. Hueston WJ. Preterm contractions in community settings: II. Predicting preterm birth in women with preterm contractions. Obstet Gynecol 1998; 92: 43-46.
3. King JF, Grant A, Keirse MJ, Chalmers I. Betamimetics in preterm labour: An overview of the randomized clinical trials. Br J Obstet Gynaecol 1988; 95: 211-222.

4. IAMS JD. Prediction and early detection of preterm labor. Obstet Gynecol 2003; 101: 402-412.

5. Crane JMG, Van den Hof F, Armson BA, Liston R. Transvaginal ultrasound in the prediction of preterm delivery: Singleton and twin gestations. Obstet Gynecol 1997; 90: 357-363.

6. Leitich H, Brumbauer M, Kaider A, Egarter C, HussLein P. Cervical length and dilation of the internal os detected by vaginal ultrasonography as markers for preterm delivery: A systematic review. Am J Obstet Gynecol 1999; 181: 1465-1472.

7. Protocolos SEGO: Amenaza de parto prematuro 2004.

8. Martín M, Cabrillo E, Carreras E. Amenaza de parto prematuro y rotura prematura de membranas. En: Urgencias en Ginecología y Obstetricia: Aproximación a la Medicina basada en la evidancia. Cañete ML, Cabero L. Ed FISCAM, 2007: 128-129; 129-132; 134-135.

9. JoHNSON JR, IAMS JD. Cervical insufficiency. En: UpToDate, Rose, BD (Ed), UpToDate, Waltham, MA, 2008.

10. To MS, Alfirevic Z, Heath VC, Cicero S, Cacho AM, Wiliamson PR et al. Cervical cerclage for prevention of preterm delivery in women with short cervix: randomised controlled trial. Lancet 2004; 363: 1849-1853.

11. Daskalakis G, Papantoniou $\mathrm{N}$, Mesogitis $\mathrm{S}$, ANTSAKLIS A. Management of cervical insufficiency and bulding fetal membranes. Obstet Gynecol 2006; 107: 221-226.

12. ACOG Practise Bulletin. Cervical insufficiency. Obstet Gynecol 2003; 102: 1091-1099.

13. Arabin B, Halbesma JR, York F, Hübener M, Van EYcK J. Is treatment with vaginal pessaries: an opcion in patients with a sonographically detected short cervix? J Perinat Med 2003; 31: 122-133.

14. Lombardía Prieto J, Fernández Pérez M. Amenaza de parto pretérmino. En: Ginecología y Obstetricia. Manual de consulta rápida. 2nd ed. TEDEC MEIJI. Editorial médica Panamericana 2007: 376.

15. Thomas McElRath MD. Midtrimester preterm premature ruptura of membranas. En: UpToDate, Rose, BD (Ed), UpToDate, Waltham, MA, 2008.

16. Protocolos SEGO: Rotura prematura de membranas 2003.

17. Lombardía Prieto J, Fernández Pérez M. Corioamnionitis. En: Ginecología y Obstetricia. Manual de consulta rápida. 2nd ed. TEDEC MEIJI. Editorial médica Panamericana 2007: 383-385. 
